\title{
EDITORIAL COMMENTS ON THE SYMPOSIUM ON MICROSCALE TEXTURES OF MATERIALS 21-23 OCTOBER 1991, CINCINNATI
}

Microstructure refers to any of the myriad features observed in sectioned samples of solid materials. Such observations are generally aided with sophisticated instruments, such as the optical microscope, or the scanning or transmission electron microscope. Contrast observed by such instruments reflects projections of the spatial distribution of component phases (and/or lattice orientations) in the volume, and in some cases aspects of the defect structure.

What is observed is never the complete microstructure of the material. Probing always disturbs the microstructure to some degree or another. Instruments are limited in resolution. Sectioning procedures are tedious and sensitive to many parameters of the preparation. As a consequence we must infer information about the total microstructure from observations taken from rather limited volumes. In many instances these observations are so limited that we cannot properly conduct statistical analysis to establish their credibility as representative of the aggregate microstructure. The projective nature of experimental observations sometimes leaves us with a complicated deconvolution, relying upon principles of stereology to extract some three-dimensional aspects of microstructure from one- or two-dimensional projections. There are, indeed, many experimental limitations in observing microstructures.

An equally difficult situation is apparent when we seek to represent experimental data. With an increasing ability to capture the details of microstructure in modern instruments, we are increasingly confronted with massive measurement sets obtained in multidimensional spaces. The early work of modern texture analysis confronted the topological challenges inherent to three-dimensional representation of the orientations of the crystal lattice (e.g. Euler space). Such representations are multiconnected and topologically complex. When faced with the challenge of connecting texture analysis with spatial aspects of microstructures we are now faced with spaces of even higher dimension. For example, two-point statistics of lattice orientation, which carry first-order spatial information, reside in a nine-dimensional space. Local macroscopic description of grain boundaries requires a five-dimensional space when boundary orientation relative to the lattices is considered to be an essential parameter. These higher-order spaces present significant challenges in representation. Just communicating what is observed in experiments becomes difficult.

Notwithstanding these many challenges there has been considerable recent progress in the techniques of microscale diffraction as applied to quantitative measurements of microstructure in polycrystalline materials. The Symposium on Microscale Textures of Materials was organized in September of 1990 at the Ninth International Conference on Textures of Materials (ICOTOM 9) held in Avignon, France. It was recognized that rapid progress in microscale texture 
analysis and in its industrial applications warranted a forum for discussion of the latest results from the international community. The Texture and Anisotropy Committee of ASM International agreed to host the symposium, which was held in October 1991 in Cincinnati. Response to this forum was outstanding. The program included five technical sessions, which encompassed 23 technical papers and two round-table discussions. These sessions were very well attended, with approximately 80-90 people at peak times. During the roundtable discussions, which considered both experimental technique and questions of representation, there developed a lively debate on several issues. Some of these issues are now briefly mentioned.

\section{COMPARISONS BETWEEN VARIOUS MICRODIFFRACTION TECHNIQUES}

Throughtout the meetings there was a healthy debate of the relative merits of transmission versus scanning methods of microdiffraction. There seems to be little question, for the present time, that transmission Kikuchi diffraction methods have better spatial resolution $(\sim 0.005 \mu \mathrm{m})$ compared to the scanning methods $(\sim 0.5 \mu \mathrm{m})$. Transmission microscopy can easily be combined with scanning of samples to directly correlate local orientation with observed spatial features.

By comparison the Backscattered Kikuchi Diffraction (BKD) method (also commonly known as the Electron Back-Scattered diffraction Pattern technique) of scanning electron microscopy has the advantage that sample preparation is comparatively simple, and that large areas (volumes) can be observed in a single sample. Since only one stress free surface is introduced by sectioning, there is less tendency for surface effects in the microstructure as compared with the thin foils required in transmission microscopy. The BKD technique can be coupled with traditional scanning observations of the microstructure, such as backscattering contrast, to correlate local orientation with spatial features.

Another mode of spatial correlation is facilitated by impressive progress in automated indexing of BKD data by image analysis. Figure 1 shows some details of a set microdiffraction data obtained from $40 \%$ deformed as-cast 1100 aluminum. A "stained glass' technique has been utilized to highlight the lattice orientations of grains which contribute to elements of the preferred orientation which develops upon deformation. The colors correlate with the orientation distribution function as shown in Figure 2. (These views obtain from scanning on a hexagonal grid with $30 \mu \mathrm{m}$ spacing. Gradation of color within the grains reflects the degree of disorientation from the peak value. What is clearly evident, even at this scale, is the breakup of the grains into subgrains.) At the present time such microdiffraction analysis is achieved at rates approaching 2,000 per hour, facilitating data sets with as many as $1 \times 10^{6}$ measurements over many thousands of grains.

It is evident that both scanning and transmission methods will continue to be developed in the near future. Certainly there is a convenient merging of the length scales conveniently accessed by these two techniques which cover a vast range of microstructural features of interest. It seems that the recent gains in automated indexing applied to scanning microscopy could be implemented to a 


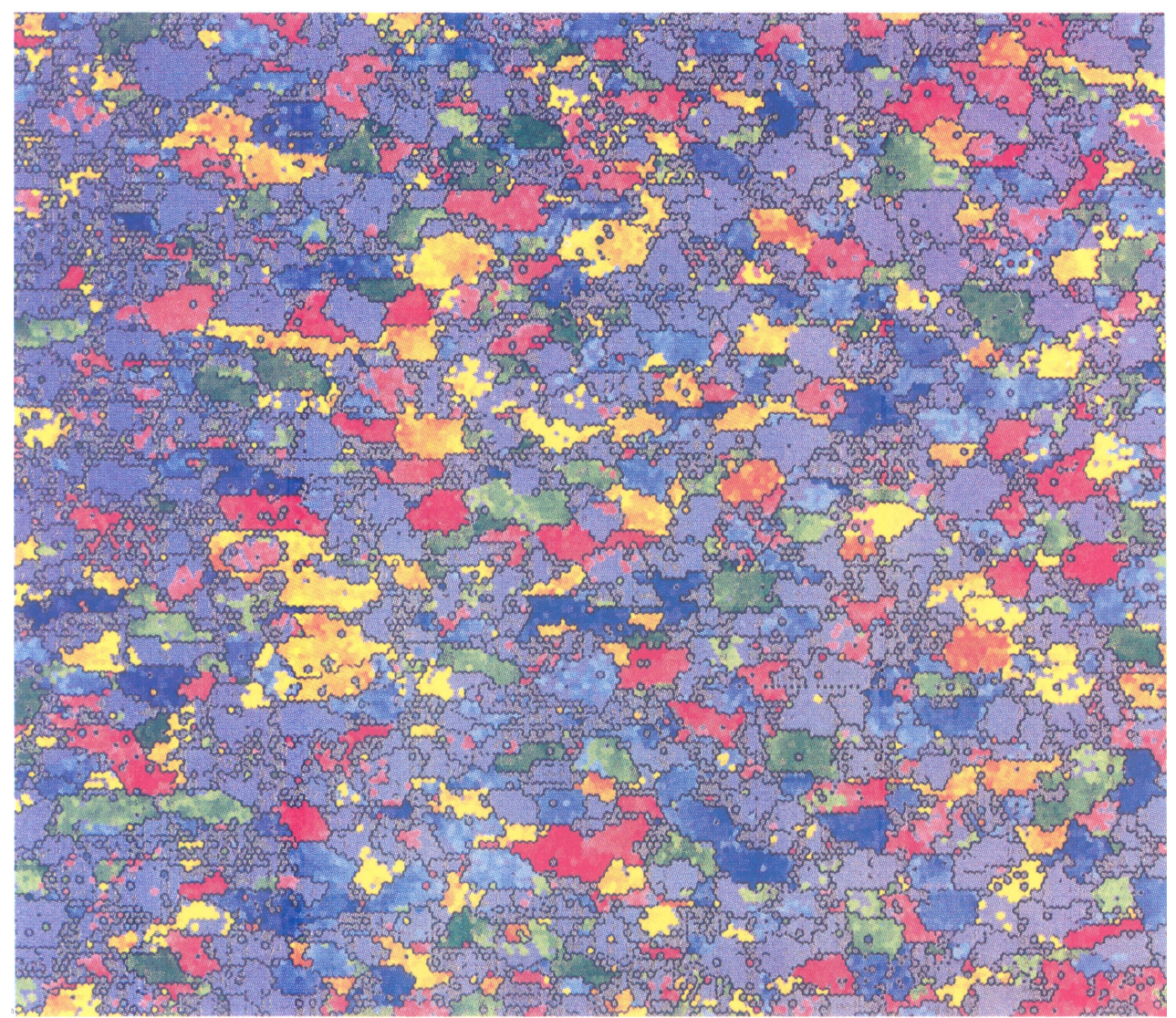

$400.0 \mu \mathrm{m}$

Figure 1 Electron diffractography of $40 \%$ channel-die compressed aluminum (sectioned normal to the compression direction) (courtesy of S. I. Wright). (See Color Plate.)

substantial degree in the realm of transmission microscopy if television camera technology of satisfactory dynamic range can be identified. In both approaches automation will facilitate the acquisition of data sets with greater statistical reliability.

X-ray microdiffraction using backscattered Laue methods offer an alternative to the electron microdiffraction methods. This requires access to the intense sources of X-rays that are achieved on synchrotrons. Some progress with this method has been described in this proceedings. While the present methods cannot yet favorably compare in spatial resolution to the electron diffraction methods, it would seem highly advisable to continue to explore these methods in the future. The major challenge, of course, would be access of such facilities to the materials community. 

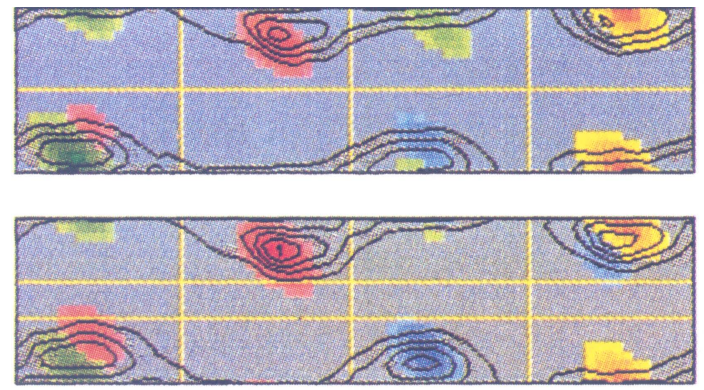

$60^{\circ}$



$65^{\circ}$



$70^{\circ}$

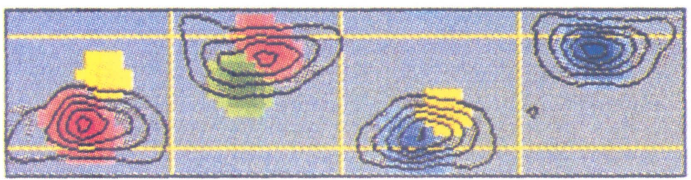

$75^{\circ}$

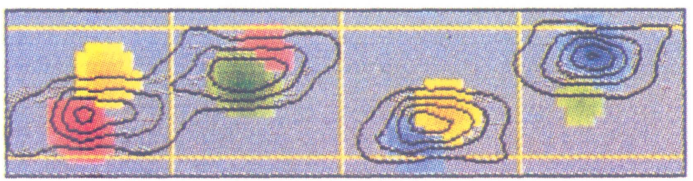

$8^{\circ}$

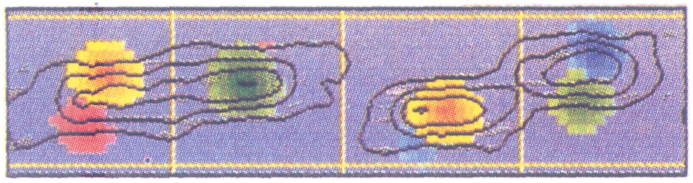

$85^{\circ}$

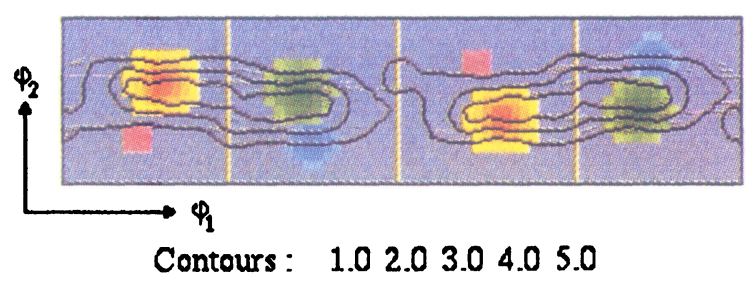

$90^{\circ}$

Figure 2 The orientation distribution function for $40 \%$ channel-die compressed commercial purity aluminum. Colors identify peak components on the $\alpha$ and $\beta$ rolling fibers. (Courtesty of S. I. Wright). (See Color Plate.) 


\section{COMPARISONS BETWEEN VARIOUS REPRESENTATIONS}

Presented at this symposium were a large number of different representations of microstructure. For example, grain boundaries were described in terms of misorientation distribution functions, focusing upon the purely rotational aspect of two lattices coming together to form a boundary. But there were differences presented in the renormalization of such representations, realizing that some natural anisotropy of misorientation distribution arises purely from the texture present in the material without the presence of any additional coherence structure. In some work the misorientation distribution was represented by classification according to the coincidence site lattice theory.

Representations of the spatial features of lattice orientation ranged from coloring schemes highlighting certain orientation or misorientation characteristics, to some highly abstract mathematical representations in various kinds of function spaces.

In all of this discussion, throughout the meetings, there was considerable duplicity, and even multiplicity, in naming representations.

It is evident that there is no consensus as to which aspects of microstructure should be represented, and how they should be communicated.

Microdiffraction clearly enables great variety in representations that can be pursued, either directly or by means of stereology. It will be a major challenge of the next decade to explore these representations and their visualizations, and to determine which shall be of greatest importance in materials research.

Brent L. Adams

Hasso Weiland

March 1992 